\title{
COMPOSIÇÃO FLORÍSTICA E ECOLOGIA DE EPÍFITAS VASCULARES NA PRAÇA CENTRAL DO MUNICÍPIO DE MAR DE ESPANHA, MINAS GERAIS, BRASIL
}

\author{
FLORISTIC COMPOSITION AND ECOLOGY OF VASCULAR EPIPHYTES AT BARÃO \\ DE AYURUOCA SQUARE, IN THE MUNICIPALITY OF MAR DE ESPANHA, MINAS \\ GERAIS, BRAZIL
}

\author{
Selma dos Santos Kaeser ${ }^{1}$, Berenice Chiavegatto ${ }^{2}$, Paulo Sergio Bordoni Ulguim ${ }^{3}$, \\ Samyra Gomes Furtado ${ }^{4}$, Luiz Menini Neto
}

\section{RESUMO}

Considerando a importância ecológica das epífitas vasculares e a escassez de estudos em áreas verdes urbanas, objetivou-se avaliar a flora epífita através do estudo da composição florística e sua estratificação vertical na praça Barão de Ayuruoca, no município de Mar de Espanha, Minas Gerais. Os parâmetros calculados foram frequências relativa e absoluta, índice de diversidade de Shannon $\left(H^{\prime}\right)$ e uniformidade de Pielou ( $(\mathcal{J}$. Foram registradas 49 espécies distribuídas em 17 famílias nas 229 árvores amostradas. As famílias mais ricas encontradas foram Bromeliaceae (nove espécies), representando $52 \%$ das ocorrências e Polypodiaceae (sete espécies) e 33\% das ocorrências. As espécies mais frequentes foram Tillandsia recurvata e T. tricholepis (Bromeliaceae) encontradas em 186 e 182 forófitos, respectivamente e Pleopeltis pleopeltifolia (Polypodiaceae) em 144. A maioria das espécies se enquadrou na categoria epífita acidental (18 espécies) e a maioria das ocorrências foi na copa das árvores. Desse modo, os resultados reforçam a capacidade de algumas espécies de Bromeliaceae e Polypodiaceae ocorrerem em ambiente com intervenção humana, além da provável interferência da perturbação antrópica nas categorias ecológicas (onde se destacam as espécies acidentais) e estratificação (com maior ocorrência na copa das árvores).

Palavras-chave: Área verde urbana; Biodiversidade; Bromeliaceae; Floresta Atlântica, Polypodiaceae.

\begin{abstract}
The aim of this work was to evaluate the epiphytic flora of an urban green area, taking into account its ecological importance and the scarcity of studies of the group in the urban environment. We evaluated the floristic composition and vertical stratification of vascular epiphytes in trees of the square Barão de Ayuruoca, in the municipality of Mar de Espanha, Minas Gerais. The calculated parameters were relative and absolute frequencies, Shannon diversity index $(H)$ and Pielou evenness $(\mathcal{J})$. We found 49 species distributed in 17 families in the 229 sampled trees. The richest families found were Bromeliaceae (nine species) and $52 \%$ occurrences and Polypodiaceae (seven species) and $33 \%$ occurrences. The most frequent species were Tillandsia recurvata and $T$. tricholepis, respectively found in 186 and 182 phorophytes, and Pleopeltis pleopeltifolia (Polypodiaceae) in 144. Most of species were classified as accidental holoepiphyte (18 species) and the majority of occurrences was in the crown of the trees. Thus, the results of this work reinforce the ability of species of Bromeliaceae and Polypodiaceae to occur in an environment with anthropic intervention, besides the probable interference of anthropic disturbance in the ecological categories, where the accidental species stand out.
\end{abstract}

Key-words: Atlantic Forest. Biodiversity; Bromeliaceae; Urban green area; Polypodiaceae..

\footnotetext{
Recebido em 25.09.2020 e aceito em 04.02.2020

1 Graduada em Ciências Biológicas. Centro de Ensino Superior de Juiz de Fora. Juiz de Fora/MG. Email: selminhakaeser@gmail.com

2 Bióloga. Doutora em Botânica. Professora do Centro de Ensino Superior de Juiz de Fora. Juiz de Fora/MG. Email: berechiavegatto@cesjf.br

3 Graduado em Ciências Biológicas. Cargo e Instituição a que pertence. Juiz de Fora/MG. Email: paulinhobordoni2013@hotmail.com

4 Bióloga. Mestre em Ecologia. Doutoranda em Ecologia na UFJF. Juiz de Fora/MG. Email: furtadosg@gmail.com

5 Biólogo. Doutor em Botânica. Professor do Departamento de Botânica da Universidade Federal de Juiz de Fora. Juiz de Fora/MG. Email: menini.neto@gmail.com
} 


\section{INTRODUÇÃO}

A constante expansão das cidades, devido ao aumento da população promove mudanças graduais em consequentemente, a expansão das cidades modifica gradualmente todos os elementos da paisagem, como o solo, a geomorfologia, a fauna, a flora, a hidrografia, o ar e o clima, criando novas paisagens, e até mesmo novos ecossistemas (BRAGA; CARVALHO, 2003). Essas mudanças têm despertado um maior interesse pela floresta urbana por parte das administrações públicas, sendo fortalecido e incentivado pela população urbana e pelo atual discurso ecológico, o qual associa às áreas verdes melhor qualidade de vida e desenvolvimento urbano (SARDINHA; CRUZ JUNIOR; SILVA, 2016).

As áreas verdes urbanas apresentam uma contribuição que vai além da paisagem e ornamentação, é também de grande importância na melhoria do microclima por meio do sombreamento, para diminuir a poluição do ar, sonora e visual, servindo, ainda, como abrigo para a fauna que vive nas cidades, além de melhorar a qualidade do meio urbano (BASSO; CORRÊA, 2014). No entanto, embora sejam muitas e evidentes as funções e benefícios das áreas verdes, sua presença ainda é pequena e os recursos para considerá-las no planejamento geral das cidades são escassos (LOBODA; DE ANGELIS, 2005).

As epífitas vasculares são plantas que se estabelecem sem conexão com o solo ou conectadas durante apenas um período da vida, usando outras plantas como suporte sem parasitá-las. Este grupo de plantas tem grande importância ecológica, sobretudo por fornecer recursos para a fauna, como frutos, néctar, pólen e água e formar micro-habitats para uma grande variedade de animais (BENZING, 1990).

Epífitas são importantes na composição da biodiversidade em florestas tropicais e representam aproximadamente $10 \%$ de toda a flora vascular, com cerca de 29.000 espécies, distribuídas em 876 gêneros e 84 famílias (ZOTZ, 2016). São representados pelas monocotiledôneas, cujas famílias e destacam-se, em termos de riqueza, as famílias Orchidaceae, Araceae e Bromeliaceae se destacam em termos de riqueza entre as angiospermas, sendo e entre as samambaias as famílias Polypodiaceae, Aspleniaceae e Hymenophyllaceae (ZOTZ, 2016).

As epífitas desenvolvem alta diversidade em pouco espaço físico (NIEDER; PROSPERI; MICHALOUD, 2001) aumentando assim a biodiversidade local. Isto se torna importante em ambiente urbano, visto que, geralmente, na arborização das cidades ocorre baixa diversidade de espécies, mesmo diante da variedade da flora nativa brasileira (GONÇALVES et al., 2004). Esse fato provavelmente está associado a preferência pelo uso de espécies exóticas devido ao pouco conhecimento ecológico silvicultural das espécies nativas ou até mesmo por serem tradicionalmente utilizadas na arborização (GONÇALVES et al., 2004). 
Segundo a Lei o 1.188 que regula o Plano Diretor Participativo aprovada pelo município de Mar de Espanha em dezembro de 2007, que dá diretrizes sobre proteção, conservação e melhoria do meio ambiente, deve-se manter a arborização de locais públicos, como ruas e praças, além de proporcionar a arborização de novos ambientes, priorizando espécies nativas (BRASIL, 2007). Ainda assim, há escassez de estudos relacionados à biodiversidade do município.

Assim, considerando a importância ecológica das epífitas vasculares e a escassez de estudos do grupo no ambiente urbano, objetivou-se avaliar a composição florística de epífitas em ambientes urbanos, avaliando a composição florística e estratificação vertical de epífitas vasculares em árvores da praça central do município de Mar de Espanha, Minas Gerais.

\section{MATERIAL E MÉTODOS}

O estudo foi realizado na praça Barão de Ayuruoca, situada a -2151'58.7"S 4300'38.0"W, na região central do município de Mar de Espanha, Minas Gerais (Figura 1), entre julho de 2017 e julho de 2018.

O município localiza-se na Zona da Mata Mineira. Apresenta uma área de $371,76 \mathrm{~km}^{2}$, altitudes que variam de 515 m (na foz do Córrego da Lagoa) a 1.063 m (no Pico dos Cocais), e o clima é caracterizado como Tropical de Altitude, com temperatura anual entre $15,4^{\circ}$ e $23,7^{\circ} \mathrm{C}$ (EDUARDO et al., 2010).

Os dados foram obtidos em todas as árvores e plantas arborecentes, pertencentes às famílias Arecaceae, Pandanaceae, Strelitziaceae, etc., presentes na área da praça, considerando as que apresentaram diâmetro a altura do peito (DAP) superior a $5,0 \mathrm{~cm}$. As espécies encontradas foram avaliadas, registradas por meio de fotos e os dados anotados e posteriormente organizados em planilhas para serem analisados.

As espécies de epífitas encontradas foram classificadas, por sua relação com o forófito, nas categorias ecológicas propostas por Benzing (1990), sendo elas: holoepífitas características (HLC), holoepífitas facultativas (HLF), epífitas acidentais (EA) e hemiepífitas (HEM). 

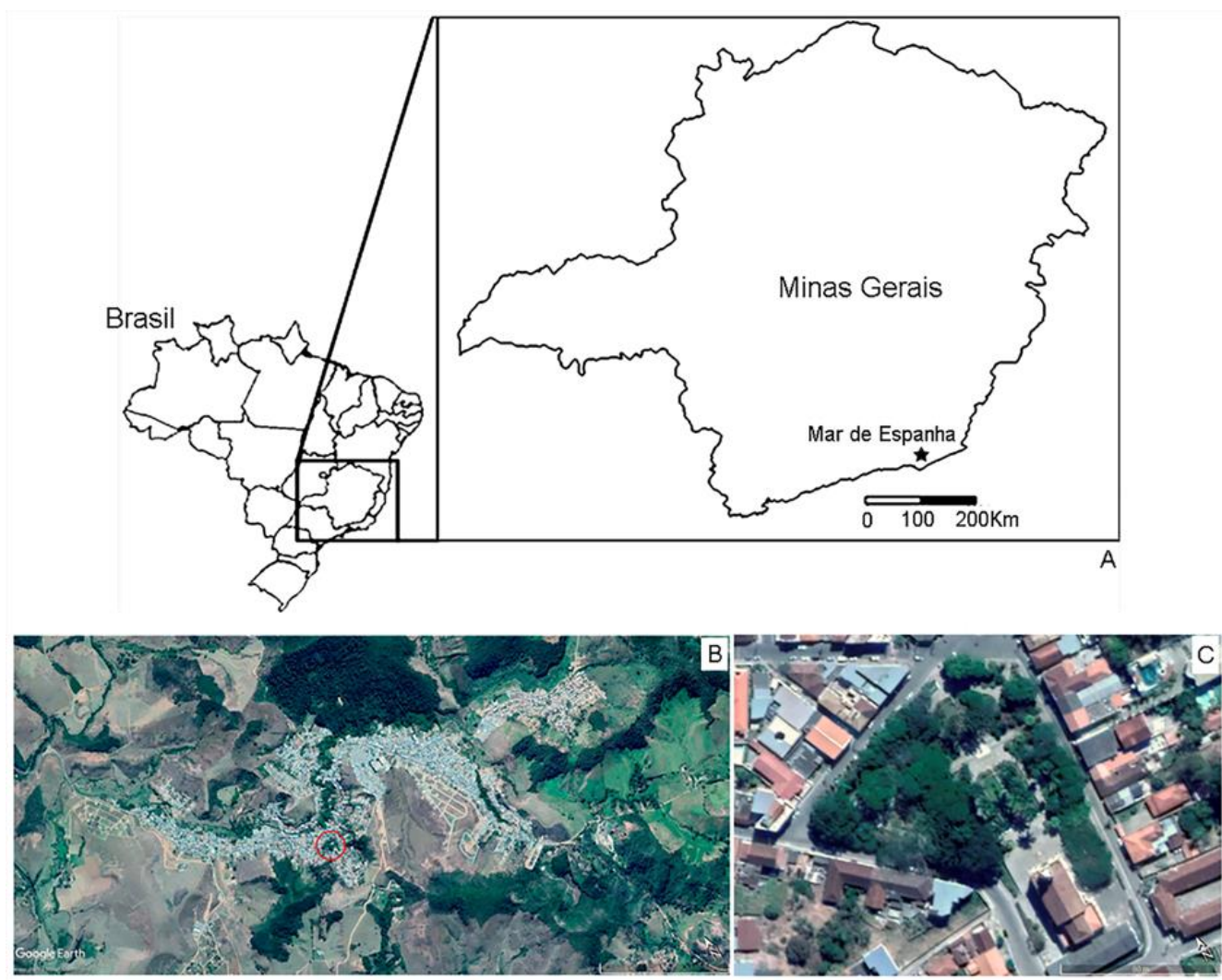

Figura 1. Localização da área de estudo: A - Localização de Mar de Espanha; B - município de Mar de Espanha destacando a área de estudo; C - detalhe da Praça Barão de Ayuruoca.

Figure 1. Location of the study area: A - Location of Mar de Espanha; B - municipality of Mar de Espanha highlighting the study area; $\mathrm{C}$ - detail of Barão de Ayuruoca square

Para a análise da distribuição vertical das espécies, os forófitos foram identificados e divididos em três estratos: 1- metade basal do tronco; 2- metade apical do tronco; 3- copa; e registradas com base na presença/ausência de espécies. Nos forófitos que não apresentavam ramificação do caule, impossibilitando essa divisão, as espécies de epífitas foram apenas contabilizadas para o total e não incluídas nos cálculos de estratificação.

A análise dos dados foi feita calculando os parâmetros frequências relativas e absolutas e índice de diversidade de Shannon $\left(H^{\prime}\right)$ e uniformidade de Pielou (J). As análises foram feitas nos programas Microsoft Excel e Past v. 3.

\section{RESULTADOS E DISCUSSÃO}

Foram contabilizadas 229 árvores e plantas aborescentes com DAP superior a $5,0 \mathrm{~cm}$, dos quais foram identificadas 26 famílias, 34 gêneros e 41 espécies (Tabela 1), com altura entre 2,5 e 18 metros. 
As famílias com maior número de indivíduos foram Arecaceae (37\%), Rutaceae (12\%), Chrysobalanaceae (9\%), Fabaceae (7\%), Bignoniaceae (5\%), Moraceae (4\%) e as demais famílias somadas com aproximadamente $25 \%$.

Dentre as 229 árvores encontradas, apenas 16 não apresentaram nenhuma epífita vascular, representando uma frequência de forófitos pouco acima que 93\%. Para Alves et al. (2014), algumas espécies arbóreas possuem uma arquitetura que não propicia o epifitismo, assim como a presença de forófitos jovens que ainda não foram colonizados, podendo interferir negativamente no estabelecimento de epífitas.

A maior parte dos forófitos é de origem exótica, com 21 espécies (51,2\%), e 13 espécies nativas $(31,7 \%)$, com sete espécies não identificadas $(17,1 \%)$. A predominância de espécies exóticas pode estar relacionada com a tendência de se utilizar plantas trazidas de outros países na arborização de ruas e praças. Segundo Lorenzi (2002), 80\% da vegetação urbana é de origem exótica, o que interfere na manutenção do ecossistema local e pode prejudicar as espécies nativas. Segundo Oliveira Neto, Fonseca e Carvalho (2014) nem toda espécie exótica é prejudicial para o ambiente, muitas se mantêm contidas e não se tornam invasoras. Aparentemente, a alta ocorrência de forófitos exóticos não é prejudicial à ocorrência das epífitas vasculares, podendo ter um papel importante no suporte e conservação das comunidades de epífitas vasculares.

Tabela 1. Espécies de forófitos amostrados no município de Mar de Espanha, Minas Gerais, Brasil $(E=$ exótica; $\mathrm{N}=$ nativa)

Table 1. Sampled phorophyte species in the municipality of Mar de Espanha, Minas Gerais, Brazil (Fam= Family; $\mathrm{E}=$ exotic; $\mathrm{N}=$ native)

\begin{tabular}{lccccc}
\hline \multicolumn{1}{c}{ Espécies } & Nome popular & $\begin{array}{c}\text { No } \\
\text { ind. }\end{array}$ & Família & $\begin{array}{c}\% \\
\text { Família }\end{array}$ & Origem \\
\hline $\begin{array}{l}\text { Dypsis lutescens (H.Wendl.) Beentje } \\
\text { \& J.Dransf. }\end{array}$ & areca & 53 & Arecaceae & $37 \%$ & $\mathrm{E}$ \\
Murraya paniculata (L.) Jack. & murta & 27 & Rutaceae & $12 \%$ & $\mathrm{E}$ \\
Licania tomentosa (Benth.) Fritsch. & oiti & 21 & Chrysobalanaceae & $9 \%$ & $\mathrm{~N}$ \\
Roystonea oleracea (Jacq.) & palmeira imperial & 11 & Arecaceae & $37 \%$ & $\mathrm{E}$ \\
O.F.Cook & figueira & 10 & Moraceae & $4 \%$ & $\mathrm{E}$ \\
Ficus microcarpa L.f. & Ipê-roxo & 9 & Bignoniaceae & $5 \%$ & $\mathrm{~N}$ \\
Handroanthus heptaphyllus (Vell.) & rabo-de-peixe & 7 & Arecaceae & $37 \%$ & $\mathrm{E}$ \\
Mattos & alfeneiro & 7 & Oleaceae & $3 \%$ & $\mathrm{E}$ \\
Caryota urens L. & - & 6 & Fabaceae & $7 \%$ & - \\
Ligustrum lucidum W.T. Aiton. & fênix & 6 & Arecaceae & $37 \%$ & $\mathrm{E}$ \\
Fabaceae indeterminada & cedro & 5 & Meliaceae & $2 \%$ & $\mathrm{~N}$ \\
Phoenix roebelenii O'Brien. & mandacaru & 5 & Cactaceae & $2 \%$ & $\mathrm{~N}$ \\
Cedrela fissilis Vell. & dracena & 5 & Asparagaceae & $2 \%$ & $\mathrm{E}$ \\
Cereus jamacaru DC. & palmeira & 5 & Arecaceae & $37 \%$ & - \\
Dracaena sp. & & & &
\end{tabular}


Araucaria angustifolia (Bertol.)

Kuntze.

Libidibia ferrea (Mart. ex Tul.)

L.P.Queiroz.

Eudicotiledôenea Indeterminada

Poincianella pluviosa (DC.)

L.P.Queiroz.

Pachira aquatica Aubl.

Pandanus sp.

Ravenala madagascariensis Sonn.

Sapindaceae indeterminada

Triplaris americana L.

Clitoria fairchildiana R.A.Howard.

Planta morta

Spathodea campanulata P. Beauv.

Syagrus romanzoffiana (Cham.)

Glassman.

Paubrasilia echinata (Lam.) Gagnon,

H.C.Lima \& G.P.Lewis.

\begin{tabular}{|c|c|c|c|c|}
\hline araucária & 4 & Araucariaceae & $2 \%$ & $\mathrm{~N}$ \\
\hline pau-ferro & 4 & Fabaceae & $7 \%$ & $\mathrm{~N}$ \\
\hline- & 4 & indeterminada & $3 \%$ & - \\
\hline sibipiruna & 3 & Fabaceae & $7 \%$ & $\mathrm{~N}$ \\
\hline munguba & 3 & Malvaceae & $1 \%$ & $\mathrm{~N}$ \\
\hline pândano & 3 & Pandanaceae & $1 \%$ & $E$ \\
\hline árvore-do-viajante & 3 & Strelitziaceae & $1 \%$ & $E$ \\
\hline- & 3 & Sapindaceae & $1 \%$ & - \\
\hline pau-formiga & 3 & Polygonaceae & $1 \%$ & $\mathrm{~N}$ \\
\hline sombreiro & 2 & Fabaceae & $7 \%$ & $N$ \\
\hline - & 2 & indeterminada & $3 \%$ & - \\
\hline sol-da-bolívia & 2 & Bignoniaceae & $5 \%$ & $E$ \\
\hline coquinho-babão & 2 & Arecaceae & $37 \%$ & $\mathrm{~N}$ \\
\hline pau-brasil & 1 & Fabaceae & $7 \%$ & $\mathrm{~N}$ \\
\hline paineira & 1 & Malvaceae & $0,4 \%$ & $N$ \\
\hline pinheiro-japonês & 1 & Cupressaceae & $1 \%$ & $E$ \\
\hline pinheiro-português & 1 & Cupressaceae & $1 \%$ & $E$ \\
\hline flamboyant & 1 & Fabaceae & $7 \%$ & $E$ \\
\hline - & 1 & Euphorbiaceae & $0,4 \%$ & - \\
\hline grevílea & 1 & Lecythidaceae & $1 \%$ & $E$ \\
\hline sapucaia & 1 & Lecythidaceae & $1 \%$ & $E$ \\
\hline magnólia amarela & 1 & Magnoliaceae & $0,4 \%$ & $E$ \\
\hline - & 1 & Myrtaceae & $0,4 \%$ & - \\
\hline pinheiro & 1 & Pinaceae & $0,4 \%$ & $E$ \\
\hline ráfia & 1 & Arecaceae & $37 \%$ & $E$ \\
\hline castanheira & 1 & Combretaceae & $0,4 \%$ & $E$ \\
\hline chapéu-de-napoleão & 1 & Apocynaceae & $0,4 \%$ & $\mathrm{~N}$ \\
\hline
\end{tabular}

Ceiba speciosa (A. St.-Hil.) Ravenna

Cryptomeria japonica (Thunb. ex L.f.)

D.Don.

Cupressus lusitanica Mill.

Delonix regia (Hook.) Raf.

Euphorbiaceae indeterminada

Grevillea robusta A. Cunn. ex R. Br.

Lecythis pisonis Cambess.

Magnolia champaca (L.) Baill. ex

Pierre

Myrtaceae indeterminada

Pinus elliottii Engelm.

Raphia farinifera (Gaertn.) Hyl.

Terminalia catappa L.

Thevetia peruviana (Pers.) K.Schum. chapéu-de-napoleão

A espécie nativa Licania tomentosa (Benth.) Fritsch. (Chrysobalanaceae) (oiti) foi a que abrigou a maior riqueza de epífitas vasculares (oito espécies), seguido das também nativas Tabebuia heptaphylla (Vell.) Toledo (Bignoniaceae) (ipê-roxo), Araucaria angustifolia (Bertol.) Kuntze (Araucariaceae) (araucária), Cereus jamacaru DC. (Cactaceae) (mandacaru), da exótica Murraya paniculata (L.) Jack (Rutaceae) (murta) e uma espécie de Fabaceae indeterminada, todos com sete espécies cada. A composição epifítica depende das características dendrológicas das espécies de forófitos como altura, rugosidade e umidade da casca, ramificação da copa, e até mesmo características químicas (BENZING, 1990).

Foram registradas 763 ocorrências de epífitas vasculares, distribuídas em 49 espécies, 31 gêneros e 17 famílias (Tabela 2). Destacam-se as famílias Bromeliaceae com nove espécies (52\% das ocorrências), Polypodiaceae com sete espécies (33\% das ocorrências), 
Orchidaceae com cinco espécies (6\% das ocorrências) e Cactaceae com cinco espécies (4\% das ocorrências), e as demais famílias somadas representando 23 espécies e apenas 5\% das ocorrências. Araceae apresentou uma riqueza de espécies alta se comparada às demais (seis espécies), porém as ocorrências somaram apenas 1\%. Santana et al. (2017) também encontraram essas três famílias como as famílias mais ricas em um fragmento urbano em Juiz de Fora, Minas Gerais. Outros estudos comprovam que Orchidaceae, Bromeliaceae e Polypodiaceae são as famílias mundialmente mais ricas da flora epifítica (BENZING, 1990; ZOTZ, 2016). No entanto, Dislich e Mantovani (1998) destacam que as famílias Orchidaceae e Bromeliaceae, por terem grande valor ornamental e serem facilmente retiráveis das árvores, podem não apresentar um destaque tão grande devido às coletas realizadas por colecionadores. Nesse estudo, essas três famílias juntas contabilizaram $91 \%$ das espécies encontradas (Tabela 2).

Tabela 2. Espécies de epífitas registradas na praça Barão de Ayuruoca no município de Mar de Espanha, Minas Gerais, Brasil

Table 2. Species of epiphytes recorded at Barão de Ayuruoca square in the municipality of Mar de Espanha, Minas Gerais, Brazil

\begin{tabular}{|c|c|c|c|c|c|c|c|c|c|}
\hline \multirow{2}{*}{ Espécies epífitas } & \multirow{2}{*}{$\begin{array}{l}\text { № } \\
\text { for. }\end{array}$} & \multirow{2}{*}{ Fam CE } & \multirow{2}{*}{ D } & \multicolumn{2}{|c|}{ Frequência } & \multicolumn{4}{|c|}{ Estratos } \\
\hline & & & & $\mathbf{F R}$ & FA & & A & B & C \\
\hline Tillandsia recurvata (L.) L. & 186 & Bro HLC & A & 24,38 & 81,22 & $\mathrm{~N}$ & 27 & 35 & 9 \\
\hline Tillandsia tricholepis Baker & 182 & Bro HLC & A & 23,85 & 79,47 & $\mathrm{~N}$ & 23 & 35 & 93 \\
\hline Pleopeltis pleopeltifolia (Raddi) Alston & 144 & Pol HLC & A & 18,87 & 62,88 & $\mathrm{~N}$ & 7 & 18 & 6 \\
\hline Pleopeltis astrolepis (Liebm.) E.Fourn & 78 & Pol HLF & A & 10,22 & 34,06 & $\mathrm{~N}$ & 2 & 13 & 4 \\
\hline Dendrobium nobile Lindl. & 26 & Orc HLC & $A$ & 3,41 & 11,35 & $E$ & 2 & 2 & 14 \\
\hline Microgramma squamulosa (Kaulf.) de la Sota & 17 & Pol HLF & A & 2,23 & 7,42 & $\mathrm{~N}$ & 0 & 1 & 13 \\
\hline Epiphyllum phyllanthus (L.) Haw. & 14 & Cac HLC & Z & 1,83 & 6,11 & $\mathrm{~N}$ & 0 & 2 & 13 \\
\hline Oncidium baueri Lindl. & 14 & OrC HLC & A & 1,83 & 6,11 & $\mathrm{~N}$ & 1 & 1 & 11 \\
\hline Aechmea nudicaulis (L.) Griseb. & 10 & Bro HLF & $\mathrm{Z}$ & 1,31 & 4,37 & $\mathrm{~N}$ & 0 & 0 & 9 \\
\hline ramosa Mart. e> & 7 & Bro HLF & $\mathrm{Z}$ & 0,92 & 3,05 & $\mathrm{~N}$ & 0 & 0 & 6 \\
\hline Aechmea sp. & 7 & Bro HLF & Z & 0,92 & 3,06 & $\mathrm{~N}$ & 0 & 0 & 6 \\
\hline Epiphyllum oxypetalum (DC.) Haw. & 5 & Cac HLC & Z & 0,65 & 2,18 & $\mathrm{~N}^{*}$ & 0 & 0 & 4 \\
\hline Ficus sp. & 5 & Mor EA & Z & 0,65 & 2,18 & - & 0 & 1 & 3 \\
\hline Arecaceae & 5 & Are $E A$ & Z & 0,65 & 2,18 & 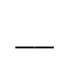 & 0 & 0 & 5 \\
\hline Pleopeltis minima (Bory) J. Prado \& R.Y. Hirai. & 5 & Pol HLC & A & 0,65 & 2,18 & $\mathrm{~N}$ & 0 & 1 & 4 \\
\hline Selenicereus anthonyanus (Alexander) D.R.Hunt. & 5 & Cac HLC & Z & 0,65 & 2,18 & $E$ & 0 & 1 & 5 \\
\hline Dendrobium moschatum (Buch. Ham.) Sw. & 3 & Orc HLC & A & 0,39 & 1,31 & $E$ & 1 & 1 & 1 \\
\hline Dichorisandra sp. & 3 & Com EA & $\mathrm{Au}$ & 0,39 & 1,31 & $\mathrm{~N}$ & 0 & 0 & 3 \\
\hline Eudicotiledônea indeterminada & 3 & Ind $E A$ & - & 0,39 & 1,31 & - & 0 & 1 & 0 \\
\hline Hylocereus setaceus (Salm-Dyck ex DC & 3 & Cac HEM & Z & 0,39 & 1,31 & $\mathrm{~N}$ & 0 & 1 & 3 \\
\hline Microgramma vacciniifolia (Langsd. \& Fisch.) Copel. & 3 & Pol HLC & A & 0,39 & 1,31 & $\mathrm{~N}$ & 1 & 1 & 3 \\
\hline Nephrolepis sp. & 3 & Nep _ & A & 0,39 & 1,31 & 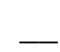 & 1 & 0 & 2 \\
\hline Schefflera sp. & 3 & Ara EA & $\mathrm{Z}$ & 0,39 & 1,31 & & 0 & 0 & 2 \\
\hline
\end{tabular}




\begin{tabular}{|c|c|c|c|c|c|c|c|c|c|}
\hline Billbergia zebrina (Herb.) Lindl. & 2 & Bro & HLC & Z & 0,26 & 0,87 & $\mathrm{~N}$ & 0 & 0 \\
\hline Ficus microcarpa L.f. & 2 & Mor & $E A$ & Z & 0,26 & 0,87 & $E$ & 0 & 0 \\
\hline Gomesa flexuosa (Lodd.) M.W.Chase \& N.H.Williams & 2 & Orc & HLC & A & 0,26 & 0,87 & $\mathrm{~N}$ & 0 & 0 \\
\hline Quesnelia testudo Lindm. & 2 & Bro & HLF & Z & 0,26 & 0,87 & $\mathrm{~N}$ & 0 & 0 \\
\hline Renanthera coccinea Lour. & 2 & Orc & HLC & A & 0,26 & 0,87 & $E$ & 0 & 0 \\
\hline Sansevieria trifasciata Prain & 2 & Asp & EA & Z & 0,26 & 0,87 & $E$ & 0 & 0 \\
\hline Acidental indeterminada & 1 & Ind & EA & 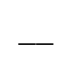 & 0,13 & 0,44 & - & 0 & 0 \\
\hline Anthurium sp. & 1 & Ara & EA & Z & 0,13 & 0,44 & $\mathrm{~N}$ & 0 & 0 \\
\hline Anthurium andraeanum Linden ex André & 1 & Ara & EA & Z & 0,13 & 0,44 & $E$ & 0 & 0 \\
\hline Bromeliaceae indeterminada & 1 & Bro & - & - & 0,13 & 0,44 & 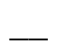 & 0 & 0 \\
\hline Cecropia sp. & 1 & $\mathrm{Cec}$ & EA & Z & 0,13 & 0,44 & $\mathrm{~N}$ & 0 & 0 \\
\hline Poaceae indeterminada & 1 & Poa & EA & A & 0,13 & 0,44 & - & 0 & 0 \\
\hline Hylocereus sp. & 1 & $\mathrm{Cac}$ & HEM & Z & 0,13 & 0,44 & $\mathrm{~N}$ & 0 & 0 \\
\hline Monstera deliciosa Liebm. & 1 & Ara & HEM & Z & 0,13 & 0,44 & $E$ & 0 & 0 \\
\hline Monstera sp. & 1 & Ara & HEM & Z & 0,13 & 0,44 & 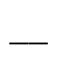 & 0 & 0 \\
\hline Oxalis sp. & 1 & Oxa & $\mathrm{EA}$ & $\mathrm{Au}$ & 0,13 & 0,44 & $\mathrm{~N}$ & 0 & 0 \\
\hline Philodendron bipinnatifidum Schott. & 1 & Ara & HEM & Z & 0,13 & 0,44 & $\mathrm{~N}$ & 0 & 0 \\
\hline Phyllanthus niruri L. & 1 & Phy & EA & $\mathrm{Au}$ & 0,13 & 0,44 & $\mathrm{~N}$ & 0 & 0 \\
\hline Pilea sp. & 1 & Urt & EA & $\mathrm{Au}$ & 0,13 & 0,44 & $\mathrm{~N}$ & 0 & 0 \\
\hline Pleopeltis hirsutissima (Raddi) de la Sota. & 1 & Pol & HLF & A & 0,13 & 0,44 & $\mathrm{~N}$ & 0 & 0 \\
\hline Serpocaulon sp. & 1 & Pol & HLF & A & 0,13 & 0,44 & $\mathrm{~N}$ & 0 & 0 \\
\hline Poincianella pluviosa (DC.) L.P.Queiroz. & 1 & $\mathrm{Fab}$ & EA & $\mathrm{Au}$ & 0,13 & 0,44 & $\mathrm{~N}$ & 0 & 0 \\
\hline Syagrus romanzoffiana (Cham.) Glassman. & 1 & Are & EA & Z & 0,13 & 0,44 & $\mathrm{~N}$ & 0 & 0 \\
\hline Syngonium angustatum Schott. & 1 & Ara & HEM & Z & 0,13 & 0,44 & $\mathrm{~N}$ & 0 & 0 \\
\hline Tradescantia zebrina Heynh. ex Bosse. & 1 & Com & $E A$ & $\mathrm{Au}$ & 0,13 & 0,44 & $\mathrm{~N}^{*}$ & 1 & 0 \\
\hline Vriesea sp. & 1 & Bro & HLF & A & 0,13 & 0,44 & $\mathrm{~N}$ & 0 & 0 \\
\hline
\end{tabular}

NOTA: № for. = número de forófitos em que a espécie foi encontrada; Fam: Famílias (Ara - Araceae, Are Arecaceae, Asp - Asphodelaceae, Bro - Bromeliaceae, Cac - Cactaceae, Cec - Cecropiaceae, Com Commelinaceae, Fab- Fabaceae, Ind - Indeterminada, Mor - Moraceae, Nep - Nephrolepidaceae, Orc Orchidaceae, Oxa - Oxalidaceae, Phy- Phyllanthaceae, Poa - Poaceae, Pol - Polypodiaceae, Urt - Urticaceae); CE = categoria ecológica $(\mathrm{HLC}=$ holoepífita característica; $\mathrm{HLF}=$ holoepífita facultativa; $\mathrm{EA}=$ epífita acidental; $\mathrm{HEM}=$ hemiepífita; $F R=$ frequência relativa; $F A=$ frequência absoluta); $D=$ forma de dispersão ( $Z=$ zoocoria; $A=$ anemocoria; $\mathrm{Au}=$ autocoria); Or. = origem $\left(\mathrm{N}=\right.$ nativa, $\mathrm{N}^{*}=$ naturalizada, $\mathrm{E}=$ exótica).

Os gêneros Tillandsia e Pleopeltis foram os que apresentaram maiores frequências absoluta e relativa (Tabela 2), representando juntos $78 \%$ das ocorrências. Tillandsia recurvata (L.) L. (Bromeliaceae) foi identificada em 186 forófitos, Tillandsia tricholepis Baker. (Bromeliaceae) em 182 forófitos, seguido de Pleopeltis pleopeltifolia (Raddi) Alston. (Polypodiaceae) em 144. Isso pode ser atribuído ao gênero Tillandsia possuir adaptações altamente xeromórficas e heliófitas, à anemocoria dessas espécies, além de ser o maior gênero de Bromeliaceae (BENZING, 1990). Tillandsia também se revelou mais frequente ou abundante em outros estudos (FABRICANTE; ANDRADE; MARQUES, 2006; FURTADO; MENINI NETO, 2015; HENRIQUES et al., 2018). Do mesmo modo, Pleopeltis, outro gênero proeminente, apresenta poiquiloidria que permite a redução de seu metabolismo quando a 
água não está disponível favorecendo sua riqueza no ambiente urbano, já que a baixa umidade não é uma barreira para seu estabelecimento e manutenção (BENZING, 1990, FURTADO; MENINI NETO, 2015).

Einzmann e Zotz (2017), destacam que as espécies epifíticas anemocóricas demonstram mais sucesso na dispersão e estabelecimento em ambientes modificados pelo homem, podendo colonizar facilmente novos habitats em paisagens fragmentadas, principalmente espécies tolerantes à seca, conforme ocorre em Tillandsia e Pleopeltis.

$\mathrm{Na}$ distribuição de espécies das epífitas segundo a categoria ecológica em relação ao forófito, verificou-se a predominância de epífitas acidentais na região estudada (18 espécies). As demais espécies foram classificadas como holoepífitas características (14 espécies), holoepífitas facultativas ( 9 espécies) e hemiepífitas (6 espécies). Esse resultado é semelhante ao encontrado por Furtado e Menini Neto (2015) e por Santana et al. (2017), que também apresentaram um alto número de epífitas acidentais, o que pode estar relacionado ao ambiente alterado por ação antrópica, com redução de competição pela ausência ou reduzida ocorrência das espécies epífitas especialistas, abrindo caminho para o estabelecimento das espécies sem adaptação ao modo de vida epifítico.

Em relação à distribuição nos estratos dos forófitos dentro dos critérios de inclusão, a maioria das espécies foram encontradas no estrato 3 (copa) com 416 ocorrências e 35 espécies $\left(H^{\prime}=2,265\right)$. Em seguida, o estrato 2 (metade apical do tronco) com 114 ocorrências e 15 espécies $\left(H^{\prime}=1,709\right)$. O estrato 1 (metade basal do tronco) foi o que apresentou menor ocorrências e número de espécies com apenas 66 e 11 respectivamente $\left(H^{\prime}=1,444\right)$. Resultados semelhantes foram encontrados por Alvim et al. (2015), onde apenas 16 espécies foram encontradas no estrato $1\left(H^{\prime}=1,52\right)$, e também por Alves e colaboradores (2014), com poucas espécies no mesmo estrato, ambos estudos também em áreas verdes urbanas.

Dentre as 49 espécies amostradas, apenas oito ocorreram nos três estratos: $D$. moschatum, D. nobile, M. vacciniifolia, O. baueri, P. astrolepis, P. pleopeltifolia, T. recurvata e T. tricholepis, o que pode ocorrer, segundo Benzing (1990) em função da variação na altura dos forófitos e da estratificação da vegetação, uma vez que a distribuição vertical das epífitas nos forófitos é determinada também por fatores como a entrada de luz e a umidade.

A luz diminui e a umidade aumenta a partir da copa até o solo da floresta, formando microclimas diferentes de acordo com a altura e a posição no forófito, possibilitando a colonização de diferentes espécies de epífitas (BENZING, 1990). Porém, isso se altera em ambientes urbanos, onde espécies de valor ornamental podem ser retiradas dos forófitos (DISLICH; MANTOVANI, 1998), o que pode ser mais fácil nos estratos inferiores. O local estudado, no entanto, aparentou-se bem cuidado pela população e servidores públicos, o que reforçado pela presença de plantas de grande valor ornamental no estrato 1 , como 0 . baueri, e 
as exóticas $D$. nobile e $D$. moschatum ressaltam a possibilidade de que impacto neste estrato ocorra pela manutenção das árvores, interferindo na distribuição. Outro aspecto a ser considerado é a maior disponibilidade de substrato na copa, como bifurcação, ramos horizontais e acúmulo de matéria orgânica para a fixação de epífitas (ALVES et al., 2014).

O índice de diversidade de Shannon $(H)$ na comunidade epifítica estudada foi de 2,092 desconsiderando as epífitas acidentais. Esse resultado é similar ao encontrado por Alvim et al. (2015) nas praças do município de Juiz de Fora, onde o valor de $H^{\prime}$ foi de 2,05 considerando as 13 praças avaliadas e 2,2 na praça mais rica do mesmo estudo e ao encontrado por Fabricante, Andrade e Marques (2006), onde $H^{\prime}$ foi de 2,371 também em árvores urbanas. De acordo com Wolf (2005), a diversidade epifítica e a densidade de indivíduos tendem a diminuir de acordo com o grau de alteração do ecossistema florestal, justificando a maior diversidade em ambientes conservados. Desse modo, o valor de $H^{\prime}$ encontrado neste estudo pode ser considerado baixo se comparado ao encontrado em ambientes mais conservados (GERALDINO; CAXAMBÚ; SOUZA, 2010; OLIVEIRA et al., 2013; PADILHA et al., 2015).

Já 0 índice de uniformidade de Pielou $(\mathcal{C}$ que avalia a participação proporcional das espécies presentes foi de 0,61. Esse baixo valor ocorre devido à grande ocorrência e, consequente dominância, das três espécies citadas acima, $T$. recurvata, $T$. tricholepis e $P$. pleopeltifolia, responsáveis também pela baixa diversidade em relação às áreas conservadas, o que pode estar relacionado à capacidade destas espécies em se estabelecer em condições adversas. Esse resultado também é similar ao encontrado por Alvim et al. (2015), onde o valor de $J$ foi de 0,588 pela dominância de quatro espécies: $T$. tricholepis, T.recurvata, $M$. squamulosa e $R$. lindbergiana.

As síndromes de dispersão mais comumente observadas foram zoocoria (22 espécies), seguido de anemocoria (17 espécies) e autocoria (6 espécies). Dettke, Orfrini e MilanezeGutierre (2008) também encontraram mais espécies zoocóricas em remanescentes perturbados, e destacam a importância dessas plantas na manutenção dos animais em ambiente urbano, os quais facilitam a dispersão de espécies para fragmentos mais próximos, representando uma importante função ecológica desempenhada pelas epífitas.

Assim sendo, a riqueza de espécies de epífitas vasculares encontrada no local de estudo pode ser considerada alta, principalmente tendo em vista o pequeno espaço físico e as perturbações do ambiente urbanizado, sendo superior ao encontrado em outros estudos realizados em Florestas Estacionais Semideciduais em diferentes estágios de conservação (FREITAS; ASSIS, 2013), destacando a importância da área estudada. 


\section{CONCLUSÕES}

Os resultados deste trabalho demonstraram a abundância e riqueza da flora epifítica na praça Barão de Ayuruoca, destacando a família Bromeliaceae em relação à riqueza de espécies. O gênero Tillandsia foi o mais registrado, e em relação à categoria ecológica, as epífitas acidentais tiveram o maior destaque, o que tem se mostrado comum em ambientes antropizados. Em relação aos forófitos exóticos e nativos, percebeu-se que ambos demonstraram ser importantes no estabelecimento das epífitas.

O presente estudo apontou um considerável número de espécies epifíticas na praça, e ressaltou sua importância ecológica em um ambiente urbano. Desse modo, os resultados obtidos nesse estudo destacam a considerável riqueza epifítica do local, principalmente em relação ao tamanho da área amostrada e ao ambiente alterado, destacando a importância de considerá-las ao pensar em estratégias de conservação, monitoramento ambiental e redução de impactos em áreas exploradas.

\section{REFERÊNCIAS}

ALVES, M. E. O.; BRUN, C., FORNO, R. S. D.; ESSI, L. Levantamento de espécies epífitas vasculares da zona urbana do município de Palmeira das Missões, RS, Brasil. Ciência e Natura, Santa Maria, v. 36, n. 3, p. 268-276, 2014.

ALVIM, F. S.; EURICO, E. S.; OLIVEIRA, M. P.; GOMES, R. L.P.; DONATO, T. P.; GONÇALVES FILHO, R. F.O; VAROTTO, Y; V.G.; FURTADO, S.G.; MENINI NETO, L.; Ecologia de epífitas vasculares em áreas verdes no município de Juiz de Fora, Minas Gerais, Brasil. Resumos expandidos. ANALECTA, Juiz de Fora, v. 1, n.1, p. 14-18, 2015.

BASSO, J. M.; CORRÊA, R. S. Arborização urbana e qualificação da paisagem. Paisagem e Ambiente: Ensaios, São Paulo, v. 1, n. 34, p. 129-148, 2014.

BENZING, D. H. Vascular epiphytes: general biology and related biota. Cambridge: Cambridge University Press, 1990, 354 p.

BRAGA, R.; CARVALHO, P. F. C. Recursos hídricos e planejamento urbano e regional. Laboratório de Planejamento Municipal-IGCE-UNESP, Rio Claro, p. 113-127, 2003.

BRASIL. Lei 1.188 de 10 de dezembro de 2007: Institui o Plano Diretor Participativo do município de Mar de Espanha-MG. Câmara Municipal de Mar de Espanha, 2007.

DETTKE, G. A.; ORFRINI, A. C.; MILANEZE-GUTIERRE, M. A. Composição e distribuição de epífitas vasculares em um remanescente alterado de floresta Estacional Semidecidual no Paraná, Brasil. Rodriguésia, Rio de Janeiro, v. 59, n.4, p.859-872, 2008.

DISLICH, R.; MANTOVANI, W. A flora de epífitas vasculares da reserva da cidade universitária "Armando de Salles Oliveira" (São Paulo, Brasil). Boletim de Botânica da Universidade de São Paulo, São Paulo, v. 17, p. 61-83, 1998. 
EDUARDO, C. C.; SILVA, A. C.; FERREIRA, C. C. M.; REZENDE, R. F.; LIMA, F. J. M. Mapeamento geomorfológico e levantamento turístico em Mar de Espanha, Minas Gerais. In: SIMPÓSIO NACIONAL DE GEOMORFOLOGIA, 8, 2010, Recife, Anais, União da Geomorfologia Brasileira, São Paulo. 1-14.

EINZMANN, H. J. R.; ZOTZ, G. Dispersal and establishment of vascular epiphytes in humanmodified landscapes. AoB Plants - Oxford Journals, Oxford, v. 9, n. 6, 2017.

FABRICANTE, J. R.; ANDRADE, L. A.; MARQUES, F. J. Componente epifítico vascular ocorrente em árvores urbanas. Cerne, Lavras, v. 12, n. 4, p. 399-405, 2006.

FREITAS, J.; ASSIS, A. M. Estrutura do componente epífitico vascular em trecho de Floresta Atlântica na região serrana do Espírito Santo. Árvore, Viçosa, v. 37, n. 5, p. 815-823, 2013.

FURTADO, S. G.; MENINI NETO, L. Diversity of vascular epiphytes in urban environment: a case study in a biodiversity hotspot, the Brazilian Atlantic Forest. CES REVISTA, Juiz de Fora, v. 29, n. 2. p. 82-101, 2015.

GERALDINO, H. C. L.; CAXAMBÚ, M. G.; SOUZA, D. C. Composição florística e estrutura da comunidade de epífitas vasculares em uma área de ecótono em Campo Mourão, PR, Brasil. Acta Botanica Brasílica, São Paulo, v. 24, n. 2, p. 469-482, 2010.

GONÇALVES, E. O.; PAIVA, H. N.; GONÇALVES, W.; JACOVINE, L. A. G. Diagnóstico dos viveiros municipais no Estado de Minas Gerais. Ciência Florestal, Santa Maria, v. 14, n. 2, p. $1-12,2004$.

HENRIQUES, L. C. M.; VICHIATO, M. R. M.; VICHIATO, M.; ÁDAMO, R. Epífitas vasculares nas Reservas Particulares Ecológicas. Tecnologia \& Ciência Agropecuária, João Pessoa, v. 12, n. 2, p. 7-13, 2018.

LOBODA, C. R.; DE ANGELIS, B. L. D. Áreas verdes públicas urbanas: conceitos, usos e funções. Ambiência - Revista do Centro de Ciências Agrárias e Ambientais, Irati, v. 1, n. 1, 2005.

LORENZI, H. Árvores brasileiras: manual de identificação e cultivo de plantas arbóreas nativas do Brasil, Nova Odessa: Editora Plantarum, 4. ed., 2002. 384p

NIEDER, J.; ROSPERIP, J.; MICHALOUD, G. Epiphytes and their contribution to canopy diversity. Plant Ecology, Burnley, v. 153, p. 51-63, 2001.

OLIVEIRA, L. C.; PADILHA, P. T.; DALMOLIN, E. B.; AZEREDO, T. E. V.; CITADINI-ZANETTE, V. Componente epifítico vascular de um fragmento florestal urbano, município de Criciúma, Santa Catarina, Brasil. Biotemas, Florianópolis, v. 26, n.2, p. 33-44, 2013.

OLIVEIRA NETO, N. E.; FONSECA, C. R.; CARVALHO, F. A. O problema das espécies arbóreas exóticas comercializadas nos viveiros florestais: Estudo de caso no município de Juiz de Fora (MG). Revista de Biologia Neotropical, Goiânia, v. 11, n. 1, p. 28-46, 2014.

PADILHA, P. T.; JUNIOR, R. S.; CUSTÓDIO, S. Z.; OLIVEIRA, L. C.; SANTOS, R.; CITADINIZANETTE, V. Comunidade epifítica vascular do Parque Estadual da Serra Furada, sul de Santa Catarina, Brasil. Ciência e Natura, Santa Maria, v.37 n.1, p. 64 - 78, 2015. 
SANTANA, L. D.; FURTADO, S. G.; NARDY, C.; LEITE, F. S.; MENINI NETO, L. Diversity, vertical structure and floristic relationships of vascular epiphytes in an urban remnant of the Brazilian Atlantic Forest. Hoehnea, São Paulo, n. 44, v. 1, p. 123-138, 2017.

SARDINHA, M. A.; CRUZ JUNIOR, F. O.; SILVA, S. K. A. As praças e arborização urbana: o caso de Macapá. Revista Científica ANAP Brasil, São Paulo, v. 9, n. 17, 2016.

WOLF, J. H. D. The response of epiphytes to anthropogenic disturbance of pine-oak forests in the higEAnds of Chiapas, Mexico. Forest Ecology and Management, Amsterdã, v. 212, p. 376-393, 2005.

ZOTZ, G. Plants on Plants - The Biology of Vascular Epiphytes. Switzerland, Springer, 2016, $282 \mathrm{p}$. 